\title{
Density-functional studies of high-pressure properties and molecular dissociations of halogen molecular crystals
}

\author{
M. S. Miao \\ Department of Physics, University of Antwerp (RUCA), Groenenborgerlaan 171, B-2020 Antwerpen, Belgium \\ and Department of Physics, Case Western Reserve University, Cleveland, Ohio 44106-7079, USA \\ V. E. Van Doren \\ Department of Physics, University of Antwerp (RUCA), Groenenborgerlaan 171, B-2020 Antwerpen, Belgium \\ José Luís Martins \\ Instituto de Engenharia de Sistemas e Computatores, Rua Alves Redol 9, 1000-029 Lisboa, Portugal \\ and Departamento de Física, Instituto Superior Técnico, Avenida Rovisco Pais, 1049-001 Lisboa, Portugal
}

(Received 17 December 2002; revised manuscript received 14 July 2003; published 4 September 2003)

\begin{abstract}
The molecular crystal structure and the molecular dissociation of iodine, bromine, and chlorine under high pressure have been studied using the pseudopotential plane-wave method with both local-density approximated (LDA) and the generalized gradient approximated (GGA) exchange-correlation functionals. Although the generalized gradient approximation overestimates the interlayer distance, it describes the geometry parameters within the molecular plane much better than the local-density approximation. In contrast to the usual geometry optimization for isolated molecules, the local-density approximation largely overestimates the bond lengths of the halogen molecules in the molecular crystal. The GGA dissociation pressures are in good agreement with the experimental values whereas the LDA dissociation pressures are lower than the experimental values. The gap closures for the three elements are observed before the dissociation pressure. The scaled parameters are universal for all three halogens in the molecular phase and at the transition points. Therefore a universal equation of state can be obtained and the dissociation pressures can be well estimated from the scaled volume at ambient pressure.
\end{abstract}

DOI: 10.1103/PhysRevB.68.094106

PACS number(s): 64.70.Kb, 71.15.Nc, 71.20.-b, 61.50.Ah

\section{INTRODUCTION}

The molecular dissociation of halogen molecular crystals under high pressure has been studied intensively by diamond-anvil cell technique. ${ }^{1-3}$ The fact that the molecular dissociations are accompanied by a metal-insulator (MI) transition makes the problem more interesting and challenging. The solid halogens are also the prototypical diatomic molecular crystals analogous to hydrogen which was predicted theoretically (but never detected experimentally) to process a molecular dissociation when densely compressed.

By using the $\mathrm{x}$-ray diffraction technique, the molecular dissociation is observed for iodine, $\mathrm{I}_{2}$, at $21 \mathrm{GPa},{ }^{3,4}$ for $\mathrm{IBr}$ at $39 \mathrm{GPa}^{5}$ and for $\mathrm{Br}_{2}$ at $82 \mathrm{GPa} .{ }^{1} \mathrm{~A}$ scaling rule was proposed by introducing an asymmetry parameter $\Delta=\left[\left(2 b_{L}\right)^{2}\right.$ $\left.+c_{L}^{2}\right]^{1 / 2} / 4 r_{s}-1$ and a scaled volume $\bar{V}=V / 8 r_{s}^{3} .{ }^{6}$ Here $r_{s}$ is the molecular bond length in solid, $b_{L}$, and $c_{L}$ are the lattice constants within the molecular plane for low-pressure phase. It might be better to use $4 \sqrt{2} r_{s}^{3}$ instead of $8 r_{s}^{3}$ to scale the volume. $4 \sqrt{2} r_{s}^{3}$ is the corresponding volume of the same unit if the crystal changes to an ideal monatomic face-centeredcubic structure whereas the nearest-neighbor interatomic distance is kept as $r_{s}$. For easier comparison with the previous experimental results, we will still use $\bar{V}=V / 8 r_{s}^{3}$ as the scaled volume. The solid halogens undergo a molecular dissociation when $\Delta$ and $\bar{V}$ reach their critical values which are assumed to be similar for all the halogens. The metallization of iodine has been reported some years ago ${ }^{7}$ and the MI transition was found at $16 \mathrm{GPa},{ }^{8,9}$ a pressure lower than that required for the molecular dissociation. Although there is no optical observation on the gap closure of bromine, the fact that the surface of solid bromine starts to reflect light at $60 \mathrm{GPa}$ indicates that the crystal reaches the metallic region around $60 \mathrm{GPa}^{10}{ }^{10}$ The extrapolation of the equation of state (EOS) for chlorine showed that the gap closure will take place at $165 \pm 30 \mathrm{GPa}$ while its molecular dissociation occurs at 220 $\pm 40 \mathrm{GPa}^{10}$

Further phase transitions were observed for the monatomic iodine crystal under pressures of 43 and $55 \mathrm{GPa}$ with the last transition to a face-centered-cubic phase. ${ }^{2,11}$ Reference 2 denoted the molecular phase as phase I, and the monatomic phases as phases II, III, and IV, respectively. As observed in Ref. 2, phase II is a body-centered orthorhombic phase with axes $a_{H}, b_{H}$, and $c_{H}$, where $H$ indicates the high-pressure phase. $a_{H}$ and $c_{H}$ are slightly different from each other but significantly different from $b_{H}$. As the pressure increases, at about $43 \mathrm{GPa}, a_{H}$ and $c_{H}$ gradually become equal and the crystal changes to body-centered tetragonal (phase III). At a pressure of $55 \mathrm{GPa}$, the system finally changes to phase IV which is face-centered cubic with $b_{H}$ $=\sqrt{2} a_{H}$.

The structural transformations of the monatomic phases have been studied by a pseudopotential method for iodine $^{12,13}$ and the pressure effects on the electronic structure and the electron-lattice interaction of fcc phase of iodine by a full-potential linearized augmented-plane-wave method. ${ }^{14} \mathrm{As}$ 
for molecular crystals, several theoretical studies have been reported including empirical models ${ }^{15-17}$ and first-principles calculations. ${ }^{14,18-20}$ The MI transition was first studied by a two-dimensional $5 p$ electron mode ${ }^{15,16}$ that gave a gap closure pressure of about $20 \mathrm{GPa}$ for iodine. The model was also generalized to three dimensions and used to study the band structure of iodine molecular crystal. ${ }^{17}$ The band overlap has also been investigated by an $a b$ initio pseudopotential study. ${ }^{18}$ The $A_{g}$ modes, the hyperfine parameters, and the band structures of both iodine and bromine molecular crystals have been studied by a full-potential linear-muffin-tinorbital method and the local-density approximation (LDA). ${ }^{19,20}$ In that work, the experimental lattice constants were adopted while the atom positions were fully optimized. However, it has been a challenging problem for a long time to obtain the molecular dissociation from first-principles calculations. This is because the energy difference between the molecular and the atomic phases is very small around the dissociation point. It is found by calculation ${ }^{19}$ that the molecular phase dissociates to an atomic face centered orthorhombic (fco) phase while the experimental lattice constants were used.

To overcome the above difficulties, we will perform a full optimization including the atomic positions and the lattice constants for both the molecular and the atomic bodycentered orthorhombic phases of iodine, bromine, and chlorine in a wide pressure range. The convergence on the energy cutoff and the $k$ mesh for Brillouin zone integrations were carefully checked. Since the energy difference between the molecular phase and the atomic phase around the dissociation point is very small, we adopt the technique of choosing equivalent $k$ points for the two phases. Using the same technique, the energy differences of the different polytypes of silicon carbide $(\mathrm{SiC})$, which are as small as several meV, have been successfully obtained. ${ }^{21}$ Besides calculating the geometry as well as the elastic properties of molecular crystals under zero and high pressures, the problems of the molecular dissociation and the band-gap closure will be particularly addressed. After a brief introduction on the methods used and the calculational procedure in Sec. II, the results will be presented and discussed in Sec. III and conclusions will be made in Sec. V.

\section{METHODS AND COMPUTATIONAL PROCEDURE}

The structural optimization in this paper is based on a variable-cell-shape ${ }^{22}$ optimization, where the enthalpy of the crystal is minimized with respect to both the lattice coordinates of the ions and the components of the metric (the dot products between the lattice vectors) of the simulation cell. We use a minimization algorithm by Davidon. ${ }^{23}$ The total energy is obtained by self-consistently solving the KohnSham equation for the electron states, using both the LDA Ceperley-Alder ${ }^{24}$ correlation potential as parametrized by Perdew-Zunger ${ }^{25}$ and the Perdew-Wang (PW92) generalized gradient approximation (GGA). ${ }^{26}$ The interaction of the valence electrons with the core electrons is described by an $a b$ initio norm-conserving pseudopotential. ${ }^{27}$ The nonlinear core corrections are used and relativistic effects are included for

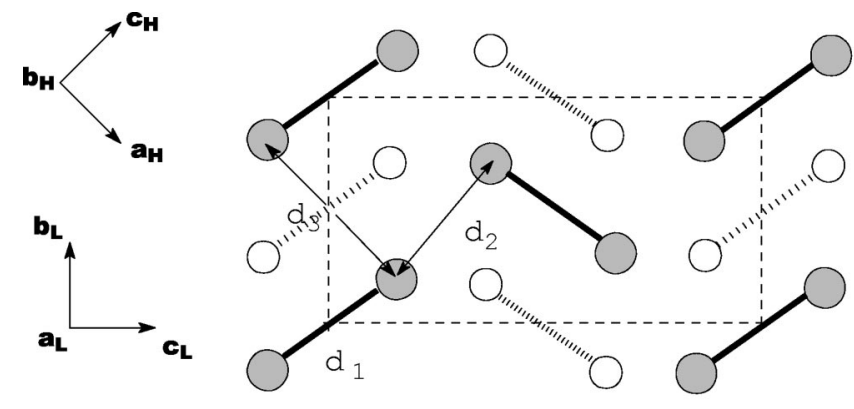

(a)

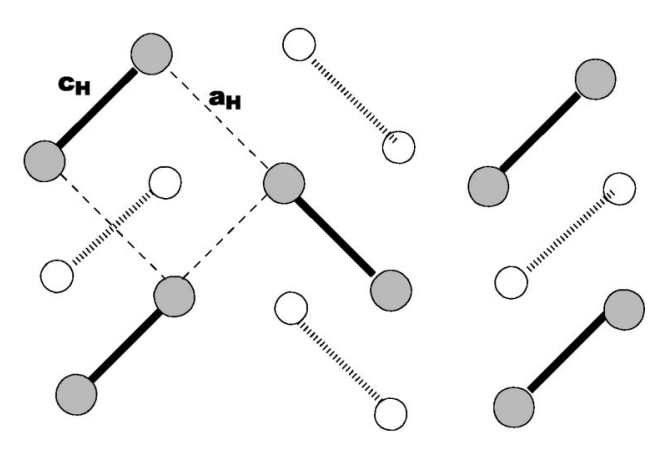

(b)

FIG. 1. The structures of halogens in molecular and monatomic phases. The dashed lines in (a) are the $b_{L}$ and $c_{L}$ axes of the molecular phase and the dashed lines in (b) are the $\mathrm{a}_{H}$ and the $\mathrm{c}_{H}$ axes of the monatomic phase.

iodine and bromine. The same corrections were tested for chlorine and it was found that they can be neglected. The electronic wave functions are expanded in terms of a planewave basis set. The cutoff radii of the pseudopotentials are $1.75 \AA, 1.95 \AA$, and $2.40 \AA$ for chlorine, bromine, and iodine, respectively. A cutoff energy of $50 \mathrm{Ry}$ is used to obtain convergence of the total energy and pressure with respect to basis set size. This large basis is needed especially for getting a good convergence on the stress. That corresponds to using around 10000 plane waves for the larger cells. A $k$ mesh of $4 \times 2 \times 4$ which corresponds to four $k$ points is used for the molecular phase calculations. For studying the molecular dissociation point, one needs to compare the total energies of the molecular and the atomic phases. Since the energy difference between these two phases around the transition point is very small, a comparable choice of the $k$ points for two phases is found necessary in practice. Thus, a conventional unit cell containing four atoms is used together with a 4 $\times 4 \times 4 k$ mesh for the atomic phase. These $k$ points are distributed similarly to those for the molecular phase in the corresponding Brillouin zones, such that the numerical error of the Brillouin zone integration will be minimized.

Figure 1 emphasizes the close relationship between phases I-IV. In Fig. 1(a) we show the low-pressure structure of halogens, phase I, which is base-centered orthorhombic $^{28,29}$ (space group, $V_{h}^{18}$ ) with four (two) diatomic molecules in the conventional (primitive) unit cell. It is 
drawn in projection with respect to the $a_{L}$ axis (the index $L$ is for low pressure), atoms and bonds drawn with solid lines are in the basal $b_{L}-c_{L}$ plane, those drawn with dashed lines are in a plane with a height of $a_{L} / 2$. In the $b_{L}-c_{L}$ plane, we have a short interatomic distance $d_{1}$ corresponding to the bond within the $X_{2}$ molecules. In the figure, we also identify the second and the third neighbor interatomic distances between atoms from different molecules in the same plane, $d_{2}$ and $d_{3}$. In Fig.1(b) we show the structure of phase II, which is body-centered orthorhombic with two (one) molecules in the conventional (primitive) unit cell. It is drawn in projection with respect to the $b_{H}$ axis (the index $H$ is for high pressure). Again we draw the atoms and bonds in the basal $a_{H^{-}} c_{H}$ plane with solid lines, and those drawn with dashed lines are in a plane with a height of $b_{H} / 2$. The thick bond lines do not show the shortest bonds, they just emphasize the relationship with phase I. For the special case $a_{H}=c_{H}$, the structure becomes body-centered tetragonal, which is phase III. If furthermore $b_{H}=\sqrt{2} a_{H}$, the structure becomes facecentered cubic, which is phase IV. Notice that for the special case of $2 c_{L}=b_{L}$ and for $d_{1}=d_{2}=d_{3}$ phase I becomes the same as phase III, with $\sqrt{2} c_{H}=c_{L}$.

In our relaxation of the crystal structure the symmetry of the system is preserved. Changes to a higher symmetric structure are permitted but the inverse procedure, symmetry breaking, is not allowed as the calculated forces and stresses have the symmetry of the system and the minimization algorithm does not break that symmetry (see Ref. 30 for the details).

\section{RESULTS AND DISCUSSION}

\section{A. Geometries under zero pressure}

The geometries of the iodine, bromine, and chlorine molecular crystals are optimized at the zero external pressure using both GGA and LDA. The results are listed in Table I together with the experimental values. From the table, it can be found that there is a systematic underestimates of 10$20 \%$ on the total volumes of all three kinds of halogens for LDA calculations. The corresponding lattice constants are also reduced for $2-15 \%$ by LDA. This is not a surprising result since LDA always underestimates the interatomic distances, no matter whether the atoms are bonded or not. All the three lattice constants are underestimated including constants $b$ and $c$, which are in the molecular plane and have a larger tendency to be reduced by LDA. It is also worth noting that in our LDA results the molecular bond lengths are larger than the experimental values, which is the opposite of the known trend for isolated molecules. This is caused by the significant reduction of the intermolecular distances which weakens the covalent intramolecular bond, giving a larger equilibrium bond length. Calculations of an isolated $\mathrm{I}_{2}$ and an isolated $\mathrm{Br}_{2}$ molecule in a large unit cell using LDA give bond lengths of $2.655 \AA$ and $2.263 \AA$ that are about $5 \%$ smaller in comparison with the experimental values of 2.67 $\AA$ and $2.28 \AA$.

GGA is known to improve the geometries of molecules as well as solids. But for weakly bound molecular systems, it is
TABLE I. The calculated and the experimental parameters of iodine, bromine, and chlorine under zero external pressure. Volumes are in $\AA^{3}$, lattice constants and the interatomic distances are in $\AA$. $d_{1}$ is the nearest-neighboring distance, i.e., bond length. $d_{2}$ as well as $d_{3}$ are the interatomic distances between the neighboring molecules. They are defined visually in Fig. 1. $\Delta$ is dimensionless and for its definition see text. The experimental data are from Wyckoff (Ref. 28).

\begin{tabular}{lcccccccc}
\hline \hline \multicolumn{1}{c}{$V$} & $a$ & $c$ & $b$ & $d_{1}$ & $d_{2}$ & $d_{3}$ & $\Delta$ \\
Iodine & & & & & & & & \\
\hline LDA & 292.68 & 7.122 & 9.579 & 4.290 & 2.80 & 3.24 & 3.66 & 0.148 \\
GGA & 357.21 & 8.522 & 9.789 & 4.543 & 2.84 & 3.37 & 3.87 & 0.176 \\
Expt. & 327.2 & 7.136 & 9.784 & 4.686 & 2.72 & 3.56 & 4.05 & 0.264 \\
Bromine & & & & & & & \\
LDA & 213.09 & 5.997 & 8.388 & 3.894 & 2.37 & 2.92 & 3.34 & 0.207 \\
GGA & 318.72 & 6.753 & 8.412 & 4.292 & 2.35 & 3.14 & 3.66 & 0.291 \\
Expt. & 260.57 & 6.67 & 8.72 & 4.48 & 2.27 & 3.31 & 3.79 & 0.377 \\
Chlorine & & & & & & & \\
LDA & 180.44 & 6.111 & 7.953 & 3.701 & 2.06 & 2.82 & 3.35 & 0.319 \\
GGA & 253.86 & 7.126 & 8.204 & 4.342 & 2.03 & 3.18 & 3.81 & 0.426 \\
Expt. & 230.91 & 6.24 & 8.26 & 4.48 & 2.02 & 3.34 & 3.70 & 0.508 \\
\hline \hline
\end{tabular}

not clear if GGA is an improvement. It was known that GGA gives no binding for polyethylene crystal, although the inchain parameters are in better agreement with the experimental values than LDA. ${ }^{31,32}$ The halogen molecular crystals are more complex. The interatomic distances between the molecules in the molecular plane $(2.80 \AA, 3.24 \AA$, and $3.66 \AA$ for iodine) are all shorter than twice the van der Waals radius (4.30 ̊ for iodine), ${ }^{4}$ which indicates a very strong intermolecular interaction. On the other hand, the molecule planes are bound by the dispersion forces. Our calculations show that PW92 GGA systematically improves the results for the bonded systems or the strongly interacting molecules. But for systems bound by van der Waals interaction, which is a problem neither LDA nor GGA is designed for, GGA tends to overestimate the length scale.

It can be seen from Table 1 that the GGA values of $b$ and $c$ are in very good agreement with the experimental values, all with a difference smaller than 5\%. The GGA bond lengths are less than 5\% larger than the experimental values. More importantly, the GGA interatomic distances between the molecules are evidently larger than LDA values, although they are still about $10 \%$ smaller than the experimental ones. A slightly larger bond length than the experimental one is a well-known feature of GGA. The PW92 GGA bond lengths for isolated $\mathrm{I}_{2}$ and $\mathrm{Br}_{2}$ molecules are found to be $2.683 \AA$ and $2.275 \AA$ that are larger than LDA results and are in very good agreement with experimental values.

However, it can be seen from Table 1 that the PW92 GGA overcorrects the LDA volumes and predicts unit cell volumes that are 10-20\% larger than experiment. Furthermore, it can be found that the main differences between the PW92 GGA and the experimental volumes are due to the overestimation of the lattice constant $a$ (interplanar distance) for which the GGA predicts values that are always $20 \%$ larger than the experimental one. To study the origin of this overestimation, 


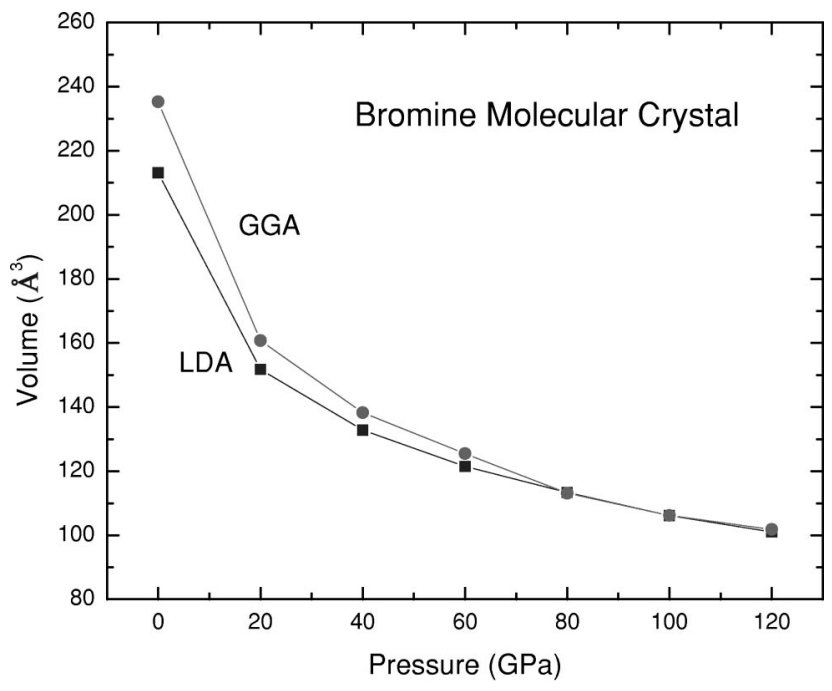

FIG. 2. The GGA and LDA volumes of a bromine molecular crystal vs the external pressure.

we calculated the GGA binding energy between the molecular planes and found a very small but positive binding energy (several $\mathrm{meV} / \mathrm{molecule}$ ) for all three halogens. The small binding energies indicate a very flat energy surface that cause large uncertainties on lattice constant $a$. Indeed, while we impose a very small external pressure, $0.2 \mathrm{GPa}, a$ changes drastically and becomes close to the experimental values. In contrast to the interplane binding, the bindings between the molecules in the molecular plane are rather large. The GGA binding energies for Bromine molecular crystals are 133 $\mathrm{meV} / \mathrm{molecule}$ inside the molecular planes and only $5 \mathrm{meV} /$ molecule between the planes.

\section{B. Geometries under high pressures and the equations of state}

A series of geometry optimizations were performed for all three halogen molecular crystals under different external pressures. The pressure steps are chosen to be 5, 20, and 50 GPa for iodine, bromine, and chlorine, respectively. These pressure steps are about $p_{c} / 4$ for each halogen crystal. Figure 2 shows the GGA and LDA volumes of the bromines molecular crystal under a series of pressures ranging from 0 $\mathrm{GPa}$ to $120 \mathrm{GPa}$. It needs to be noticed that the volume at 0.2 $\mathrm{GPa}$ instead of that at $0 \mathrm{GPa}$ is used for GGA because of the binding problem of GGA (see Sec. III A). The figure shows that although the GGA volumes at ambient pressure are much larger than the LDA ones, GGA and LDA tend to give similar volume under high pressure. At zero or low external pressures, the intermolecular bonding is weak and the charge density varies largely between the molecules, so that the gradient corrections are more important. Under high pressures, the bonding is dominated by strong chemical interactions and the charge density becomes more homogeneous. Thus, the differences between the predictions of two methods are small. The changes of the lattice constants versus pressure for bromine are presented in Fig. 3. Again the values at 0.2 $\mathrm{GPa}$ are used instead of $0 \mathrm{GPa}$. The lattice vectors reduce anisotropically with increasing external pressure, which can be easily understood by considering the larger distances and

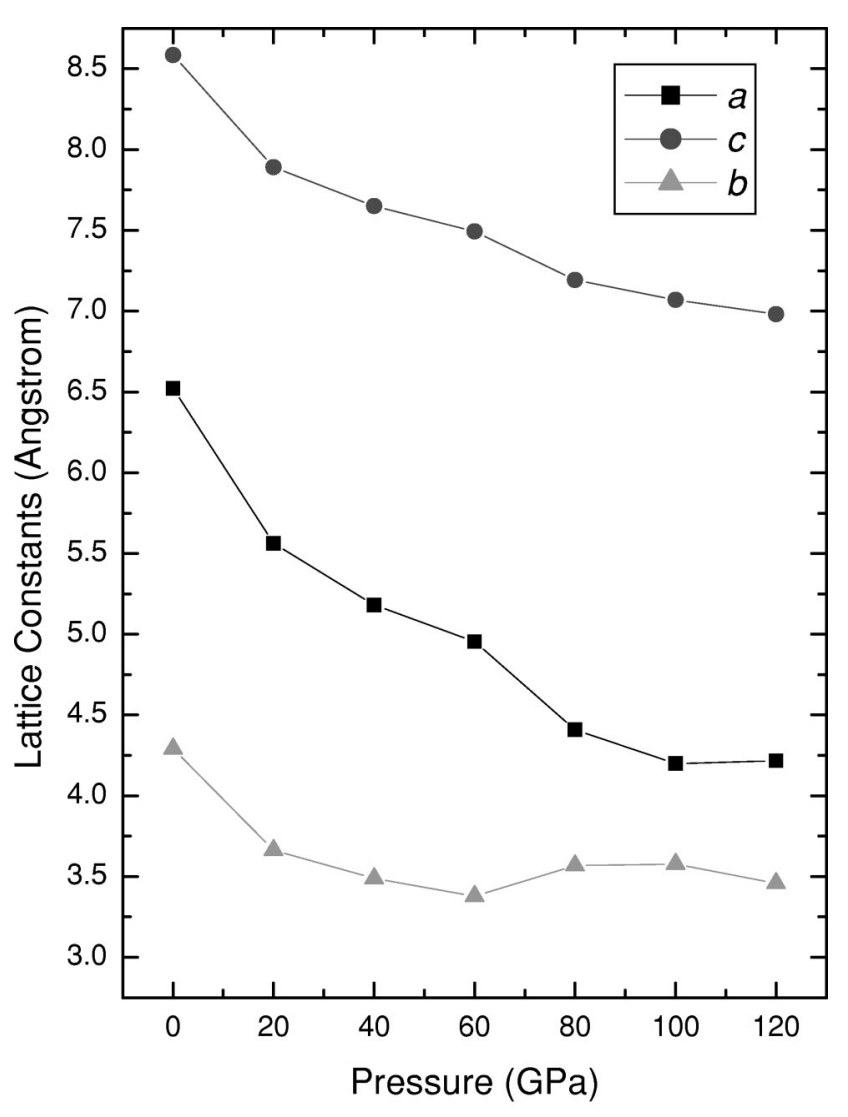

FIG. 3. The GGA lattice constants for a bromine molecular crystal under various external pressures.

the weaker interaction between the molecules in adjacent $b-c$ plane. The monotonic decrease of the constants is interrupted at $80 \mathrm{GPa}$. At that pressure, the molecular crystals change from phase I to phase $\mathrm{I}^{\prime}$ in which there is an equal interatomic distance in the molecule plane.

For showing the transition from I to I' phase, we present the GGA interatomic distances in the molecule plane for bromine in Fig. 4. At pressures less than $60 \mathrm{GPa}$, the second and the third neighboring distances decrease significantly with the increasing pressure, while the nearest-neighboring distance or, say, the bond length changes only slightly. At the pressure of $80 \mathrm{GPa}$, the relaxed structures showed that the differences between the three different interatomic distances vanish, indicating that we obtain an atomic crystal with higher symmetry. ${ }^{19}$ The above features reveal a transition from a molecular phase to a monatomic phase (phase $\mathrm{I}^{\prime}$ ). But as will be discussed in the next two subsections, this is a nonequilibrium structure as the system undergoes a firstorder phase transition to another monatomic phase (phase II) prior to I to I' transition point. It is worth noticing that the monotonic decrease of the lattice constants is interrupted by the instability between the two phases.

The total energies and the volumes are fitted to Murnaghan's equation of state. The corresponding bulk moduli and their pressure derivatives are listed in Table II for both LDA and GGA. The experimental values are obtained by fitting the $P-V$ relations presented in Fig. 3 of Ref. 1 and Fig. 5 in Ref. 10 to fourth-order polynomial, to obtain the bulk 


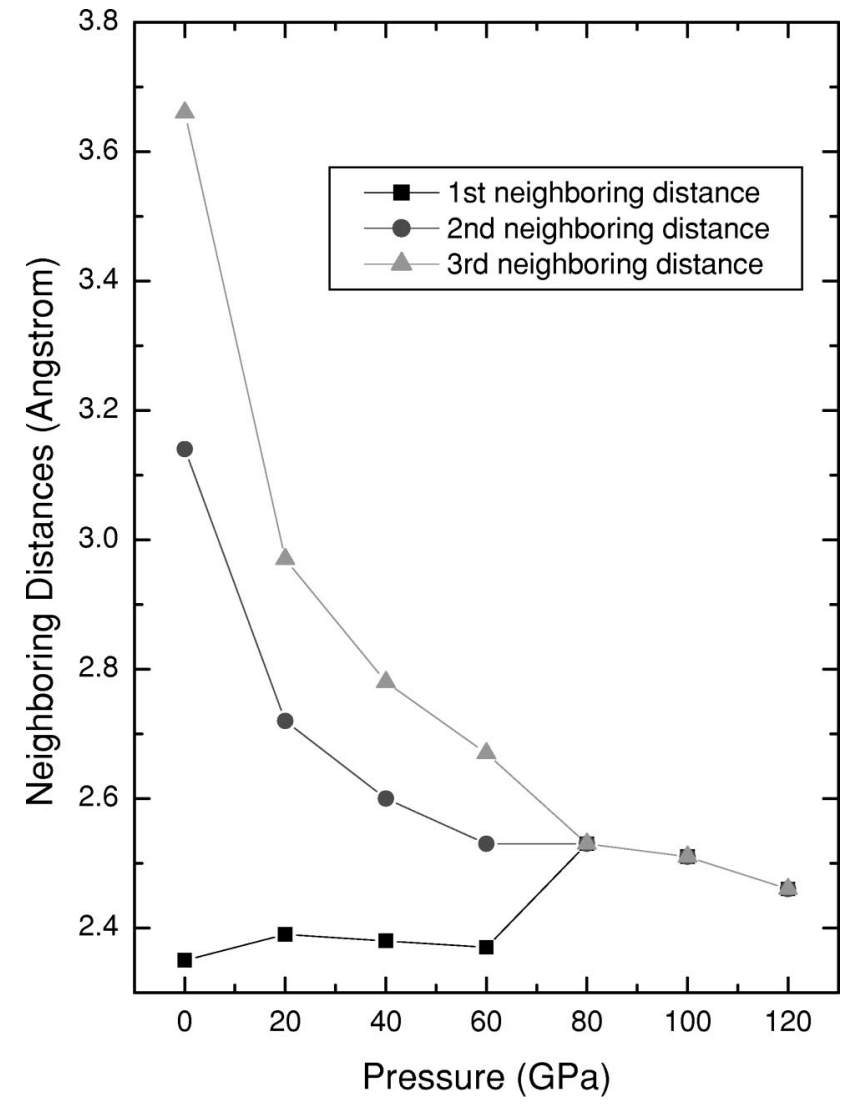

FIG. 4. The changes of the first, the second, and the third neighbor interatomic distances inside the bromine molecule plane vs the external pressure.

modulus and its pressure derivative. It can be seen that GGA bulk moduli are in better agreement with the experimental values than LDA. LDA bulk moduli for bromine and chlorine are much larger in comparison with the experiments, which is consistent to the fact that LDA intermolecular distances are smaller than those of experiments and GGA. On the other hand, the pressure derivatives of bulk moduli are all slightly larger but comparable with the experimental results. It needs to be noticed that different fitting procedures are used for experimental and calculational results. The experi-

TABLE II. The bulk modulus and their pressure derivatives for iodine, bromine, and chlorine. All moduli are in GPa.

\begin{tabular}{lccc}
\hline \hline & $\mathrm{I}_{2}$ & $\mathrm{Br}_{2}$ & $\mathrm{Cl}_{2}$ \\
\hline LDA & & & \\
$B_{0}$ & 18.1 & 18.8 & 14.5 \\
$B_{0}^{\prime}$ & 3.85 & 3.48 & 2.38 \\
GGA & & & \\
$B_{0}$ & 13.13 & 16.24 & 14.62 \\
$B_{0}^{\prime}$ & 3.40 & 3.38 & 3.22 \\
Expt. & & & \\
$B_{0}$ & 13.73 & 15.64 & 15.18 \\
$B_{0}^{\prime}$ & 2.88 & 2.21 & 1.59 \\
\hline \hline
\end{tabular}

mental data are read from the figures in Refs. 1 and 10, which will cause some uncertainties.

The values of the bulk modulus and its pressure derivative are quite universal for all three halogens. The bulk modulus is about $15 \mathrm{GPa}$ for both GGA and experimental results. The pressure derivative is about 3.4 for GGA and LDA and 2.0 for experiments. The universal bulk modulus and its pressure derivatives indicate a universal equation of state for all three halogens. Using a Murnaghan EOS, it takes the form

$$
P=\frac{B_{0}}{B_{0}^{\prime}}\left[\left(\frac{V_{0}}{V}\right)^{B_{0}^{\prime}}-1\right]=\frac{B_{0}}{B_{0}^{\prime}}\left[\left(\frac{\bar{V}_{0}}{\bar{V}}\right)^{B_{0}^{\prime}}-1\right]
$$

in which $V_{0}$ and $\bar{V}_{0}=V_{0} / 8 r_{s}^{3}$ are the original and the scaled volume at zero pressure. If the averaged GGA values of 15 GPa for $B_{0}$ and 3.3 for $B_{0}^{\prime}$ are used, we will obtain a simple universal EOS for halogen molecular crystals,

$$
P \approx 4.4\left(\frac{\bar{V}_{0}}{\bar{V}}\right)^{3.3}-4.4
$$

The equation has the same form as the Birch-Murnaghan equation and the coefficient and the component are universal to all three halogens.

\section{Transitions from phase I to II}

Figure 5 plots the LDA total energy versus volume calculated for the molecular I (and I'), monatomic II (and III), face-centered-cubic and body-centered-cubic phases of bromine. At low pressure the most stable phase is the molecular phase I. With increasing pressure phase I transforms into the monatomic phase II. At low pressure, the fcc and bcc phases have much higher energy compared with the molecular phase I and monatomic phase II. The pressure induced structural phase transition occurs along the common tangent line between the total energy curves of the two phases. The pressure of the molecular dissociation is found to be at a volume of 16 $\AA^{3}$, corresponding to a pressure of about $50-60 \mathrm{GPa}$ against the experimental value of $80 \mathrm{GPa}$. Similarly, dissociation pressures of $15 \mathrm{GPa}$ and $150 \mathrm{GPa}$ are found for iodine and chlorine, respectively.

The GGA transition pressures are $20 \mathrm{GPa}, 80 \mathrm{GPa}$, and $200 \mathrm{GPa}$ for iodine, bromine, and chlorine, respectively. They are in better agreement with the experimental values. Around the transition points, the GGA energy differences for the two phases are smaller than those of LDA. At the region near the dissociation points, phase II has lower energy by being slightly deformed from the tetragonal structure and having different atomic distances in the $a_{H}$ and $c_{H}$ directions. The optimized geometry for monatomic phases shows that GGA does not favor very much this distortion. For iodine, the largest difference between $a_{H}$ and $c_{H}$ is only $0.03 \AA$ at $35 \mathrm{GPa}$ for GGA, but is $0.2 \AA$ at $20 \mathrm{GPa}$ for LDA. For bromine these differences are $0.12 \AA$ at $100 \mathrm{GPa}$ for GGA and $0.32 \AA$ at $60 \mathrm{GPa}$ for LDA. Only for chlorine this difference is $0.21 \AA$ at $200 \mathrm{GPa}$ for GGA in comparison with 


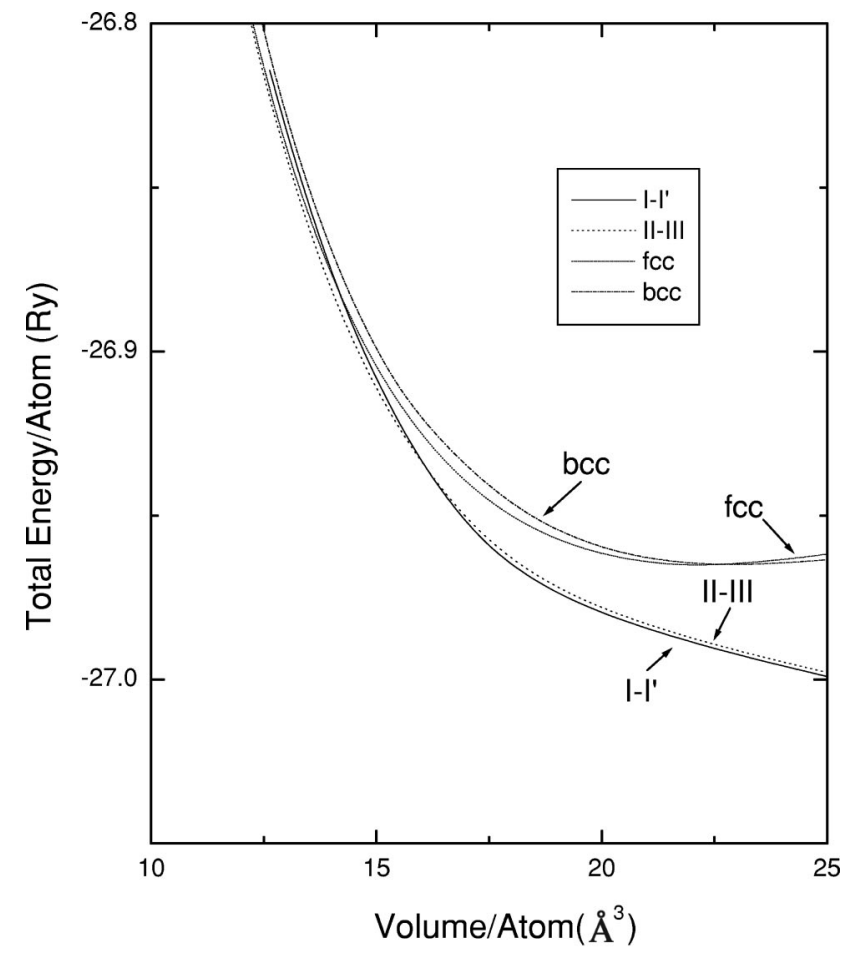

FIG. 5. Total energy vs volume of different phases of bromine. As denoted in the figure, the solid line and the dotted line are the curves of phases I and I' and of phases II and III, respectively. The dashed line and the dot-dashed line are fcc (phase IV) and bcc monatomic phases.

the value of $0.43 \AA$ at $200 \mathrm{GPa}$ for LDA. Thus, the energy differences between the low-pressure and high-pressure phases around the transition pressure become very small for GGA. For that reason, we adopt the technique of using equivalent $k$-mesh in the Brillouin zone for two different phases.

Table III shows the scaled parameters at the transition pressures from phase I to phase II for both LDA and GGA. The LDA critical values of the scaled volumes are 1.17, 1.14, and 1.12 for $\mathrm{I}, \mathrm{Br}$, and $\mathrm{Cl}$, respectively, while the GGA ones are 1.15, 1.09, and 1.07. Taking 1.2 as the universal scaled volume at the dissociation point, the transition pressure can be obtained from the universal EOS (2)

$$
P_{c} \approx 2.3 \bar{V}_{0}^{3.3}-4.4 \text {. }
$$

Using the GGA scaled volume, the above formula gives a transition pressures of $23 \mathrm{GPa}$ for iodine, $89 \mathrm{GPa}$ for bromine, and $183 \mathrm{GPa}$ for chlorine. If the experimental scaled volumes of 2.03, 2.78, and 3.50 are used, the transition pressures become $19 \mathrm{GPa}, 63 \mathrm{GPa}$, and $139 \mathrm{GPa}$, respectively. Another experimentally available example of the molecular dissociation is the iodine bromine (IBr). At low pressures, it has the same structure $\left(\mathrm{V}_{h}^{18}\right)$ as halogen elements. Its scaled volume at zero pressure is 2.4 and the dissociation pressure is $40 \mathrm{GPa}$, which fits Eq. (3) very well.

Furthermore at the dissociation point, the following relations are found for all three elements: $a_{L}: b_{L}: c_{L}: r_{s}$
TABLE III. The LDA and GGA scaled parameters at the transition pressures from phase I to phase II for iodine, bromine, and chlorine. $r_{s}$ is the bond length in the molecular crystal under ambient pressure. The scaled parameters are defined as $\bar{V}$ $=V /\left(8 * r_{s}^{3}\right), \bar{a}=a / r_{s}, b=b / r_{s}, c=c / r_{s}$, and $\Delta=\left[(2 b)^{2}+c^{2}\right]^{1 / 2} /$ $4 r_{s}-1$.

\begin{tabular}{lccccccc}
\hline \hline & $r_{s}(\AA)$ & $P(\mathrm{GPa})$ & $\bar{V}$ & $\bar{a}$ & $\bar{c}$ & $\bar{b}$ & $\Delta$ \\
\hline \multicolumn{1}{c}{ LDA } & & & & & & & \\
Iodine & 2.80 & 15 & 1.17 & 2.05 & 3.01 & 1.52 & 0.07 \\
Bromine & 2.37 & 60 & 1.14 & 2.08 & 3.20 & 1.38 & 0.05 \\
Chlorine & 2.06 & 150 & 1.12 & 2.06 & 3.17 & 1.38 & 0.05 \\
GGA & & & & & & & \\
Iodine & 2.84 & 20 & 1.15 & 2.08 & 2.98 & 1.48 & 0.05 \\
Bromine & 2.35 & 80 & 1.09 & 1.88 & 3.06 & 1.52 & 0.08 \\
Chlorine & 2.03 & 200 & 1.07 & 1.86 & 3.05 & 1.51 & 0.07 \\
Expt. & & & & & & & \\
Iodine & 2.72 & 23 & 1.29 & 2.12 & 3.38 & 1.46 & 0.11 \\
Bromine & 2.27 & 82 & 1.29 & 2.10 & 3.35 & 1.42 & 0.11 \\
Chlorine & 1.98 & $140(?)$ & & & & & $0.11(?)$ \\
\hline \hline
\end{tabular}

$\approx 2.0: 1.4: 3.2: 1$, in which $a_{L}, b_{L}$, and $c_{L}$ are the lattice constants for the molecular phase and $r_{s}$ is the molecular bond length in the crystal under ambient pressure. The order parameter $\Delta$, defined as $\left[\left(2 b_{L}\right)^{2}+c_{L}^{2}\right]^{1 / 2} / 4 r_{s}-1$ by Düsing et al. ${ }^{6}$ is found to be 0.07 for I, 0.054 for $\mathrm{Br}$, and 0.049 for $\mathrm{Cl}$ by LDA and to be 0.051 for $\mathrm{I}, 0.077$ for $\mathrm{Br}$, and 0.072 for $\mathrm{Cl}$ by GGA. These values are all small in comparison with the experimental value, 0.11 . The GGA values of $\Delta$ are larger than those of LDA except for iodine, but still smaller than the experiments. The main difference comes from the fact that the LDA and GGA molecular bond lengths are longer than the experimental results. Using the experimental bond length, a value of 0.12 is obtained for I and 0.10 and 0.09 for $\mathrm{Br}$ and $\mathrm{Cl}$ by LDA.

\section{Transitions from phase I to $I^{\prime}$}

Going to high pressure while keeping the orthorhombic symmetry as molecular phase I, the halogens finally change to a structure with equal interatomic distances $d_{1}=d_{2}=d_{3}$ in the $\vec{b}, \vec{c}$ plane (phase $\mathrm{I}^{\prime}$ ). The transition parameters are shown in Table IV. From the table, one can see that the transition pressures calculated by LDA and GGA are different. Except for iodine, the GGA transition pressures are smaller than the LDA ones, indicating that GGA favors the in-plane equal distance phase I' more than LDA does. Both $\mathrm{I}^{\prime}$ and II phases can be viewed as a deformed structure of the higher symmetric phase III. In phase III, $b_{L}=2 c_{L}=\sqrt{2} a_{H}$ $=\sqrt{2} b_{H}$. Phase II can be obtained by making a deformation along $a_{H}$ direction and phase $\mathrm{I}^{\prime}$ along $b_{L}$ direction. Starting from the molecular phase, under compression, the system gradually changes toward phase $\mathrm{I}^{\prime}$ which has equal interatomic distances, but before that it jumps to the monatomic phase II.

At the critical point of transition I to $\mathrm{I}^{\prime}$, the LDA scaled volumes are 1.08, 0.95, and 0.94 for $\mathrm{I}, \mathrm{Br}$ and $\mathrm{Cl}$. The cor- 
TABLE IV. The LDA and GGA scaled parameters at the transition pressures from phase I to phase $\mathrm{I}^{\prime}$ for iodine, bromine, and chlorine. For the definitions of the parameters, see the caption of Table III.

\begin{tabular}{lccccccc}
\hline \hline & $r_{s}(\AA)$ & $P(\mathrm{GPa})$ & $\bar{V}$ & $\bar{a}$ & $\bar{c}$ & $\bar{b}$ & $\Delta$ \\
\hline \multicolumn{1}{c}{ LDA } & & & & & & & \\
Iodine & 2.80 & 20 & 1.08 & 1.81 & 3.09 & 1.55 & 0.09 \\
Bromine & 2.37 & 120 & 0.95 & 1.91 & 3.14 & 1.27 & 0.01 \\
Chlorine & 2.06 & 250 & 0.94 & 1.88 & 2.79 & 1.42 & 0.01 \\
GGA & & & & & & & \\
Iodine & 2.84 & 30 & 1.02 & 1.86 & 3.06 & 1.25 & 0.06 \\
Bromine & 2.35 & 80 & 1.09 & 1.88 & 3.06 & 1.52 & 0.08 \\
Chlorine & 2.03 & 150 & 1.17 & 1.81 & 3.05 & 1.24 & 0.10 \\
\hline \hline
\end{tabular}

responding interatomic distances are $3.06 \AA$, $2.39 \AA$, and $2.03 \AA$, respectively. Notice that the bond length $r_{s}$ at $0 \mathrm{GPa}$ for $\mathrm{I}, \mathrm{Br}$, and $\mathrm{Cl}$ molecular crystals at ambient pressure are calculated to be $2.80 \AA, 2.37 \AA$, and $2.06 \AA$, which indicates that the scaled interatomic distances at this point are close to 1 for all three halogens. It is observed for all three halogens that at the transition point from $\mathrm{I}$ to $\mathrm{I}^{\prime}$, there is a sudden increase of the nearest-neighbor distance. A similar conclusion can be found for GGA results. The bond length, the nearest-neighboring distance, reflects the covalency of the diatomic molecule. Being compressed, the covalence of the molecules is reduced because of the stronger interactions and the overlaps between the neighboring molecules. At the transition point, the covalency is completely lost as well the molecular feature and the nearest-neighbor distances increase suddenly and become larger than the molecular bond length under ambient pressure.

\section{E. The band-gap closure and the metal-insulator transition}

The band structures of all three halogens under several different pressures are calculated and the LDA bands of bromine under $0 \mathrm{GPa}$ and under $60 \mathrm{GPa}$ are shown in Fig. 6. The bands of all three halogens show many common features. The gap is indirect along the $\Gamma-Z$ direction and reduces with increasing pressure. The gaps at the $T, Y$, and $S$ symmetric points are also reduced by the effect of pressure. In contrast, the gaps at the $S$ and $Z$ points hardly changed while the gap at the $X$ point is enlarged by the external pressure.

The changes of the gap between the valence and the conduction bands versus pressure are shown in Fig. 7 for all three halogens. For convenience, a scaled pressure $\widetilde{P}$ $=P / P_{c}$ is used. $P_{c}$ is the LDA molecular dissociation pressure. The increase of the external pressure largely widens the bands and narrows the gaps. A gap closure can be clearly seen at $10 \mathrm{GPa}$ for iodine, at $50 \mathrm{GPa}$ for bromine, and 150 $\mathrm{GPa}$ for chlorine. These indicate that a metal-insulator transition takes place before the molecular dissociation, which is in good agreement with the experimental observations. Not surprisingly, the gap closure pressures are all smaller than the experimental ones, which is due to the well known gap un-

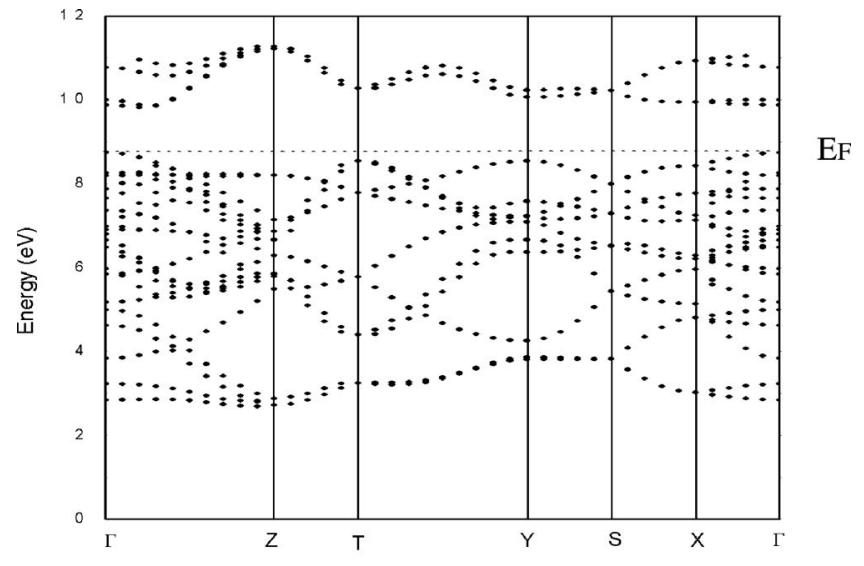

(a)

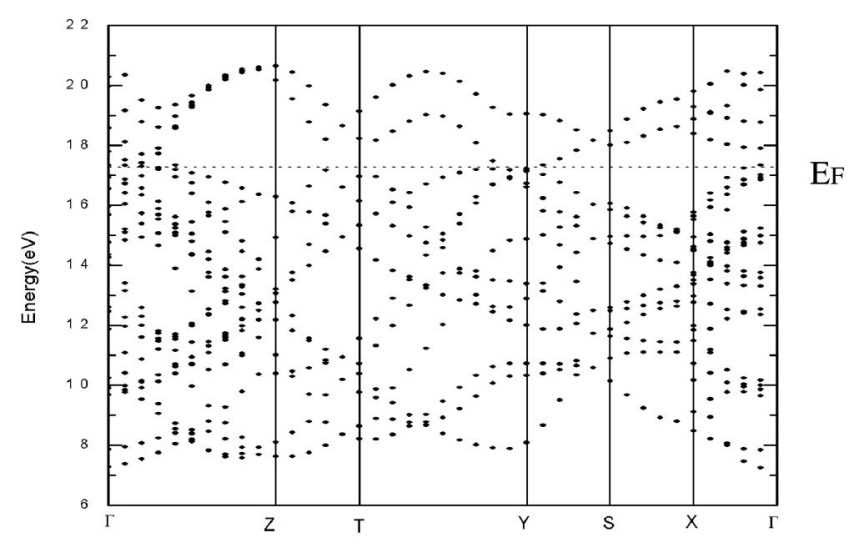

(b)

FIG. 6. The LDA bands of bromine under the external pressure of 0 [in (a)] and $60 \mathrm{GPa}$ [in (b)].

derestimation of LDA. The dependences of the energy gap on pressure are $-6.0 \times 10^{-2} \mathrm{eV} / \mathrm{GPa}$ for Iodine, -1.5 $\times 10^{-2} \mathrm{eV} / \mathrm{GPa}$ for Bromine and $-0.8 \times 10^{-2} \mathrm{eV} / \mathrm{GPa}$ for chlorine. The value for iodine is very similar to the previous

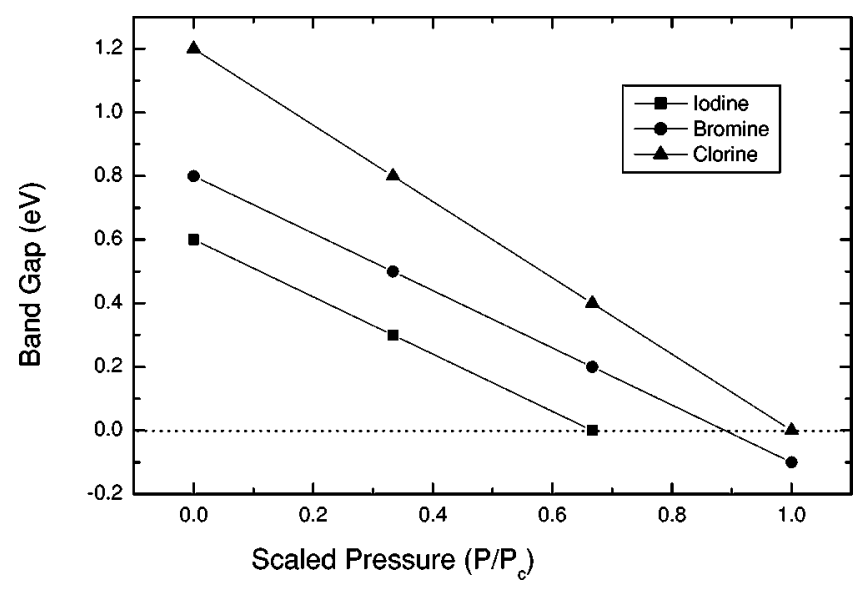

FIG. 7. The fundamental band gap vs the pressure for iodine, bromine, and chlorine. For convenience, a scaled pressure $P / P_{c}$ is used. 
$5 p$ electron tight-binding model. The LDA gap of solid iodine under $0 \mathrm{GPa}$ is about $0.6 \mathrm{eV}$, which is half of the experimental value of $1.3 \mathrm{eV}$ (Ref. 33) and the tight-binding value of $1.2 \mathrm{eV}$. The closure point of the gap for iodine was obtained to be more than $20 \mathrm{GPa}$ by tight binding. In comparison with the covalent semiconductors, the gap dependences on pressure for halogens are rather large. This is due to the losses of the covalency of the molecular bonds under high pressure.

\section{CONCLUSIONS}

In conclusion, we have performed a full geometry optimization for both the molecular and the atomic phases of three halogens: iodine, bromine, and chlorine. The convergence on the cutoff energy is carefully examined and an equivalent $k$ mesh is used for the molecular phase and the atomic phase. For the molecular phase at lower pressure, LDA underestimates all the intermolecular distances and gives a strong binding between the halogen molecules. This strong binding weakens the covalency of the bonding for the halogen molecules. Thus, LDA gives a larger bond length in contrast to its usual tendency to give shorter bond lengths for isolated molecules. The GGA in-plane parameters are in much better agreement with the experimental results. The bindings within the plane and between the planes are quite different. GGA underestimates the interplane binding energies and thus gives rise to a large uncertainty on the interplane distances. It was found that the lattice constant $a$ is dramatically overestimated by GGA.
LDA and GGA tend to give similar geometries under high pressure. As expected, the lattice constants reduce anisotropically with increasing external pressure. The scaling rule is proved to be valid in a large pressure range for all three halogens and a general equation of states has been set up with averaged GGA bulk modulus and its pressure derivative.

By using the equivalent $k$ mesh for the molecular and the atomic phases, both LDA and GGA predict a transition from the molecular phase to a monatomic phase. The dissociation pressures obtained by GGA are in good agreement with the experimental values but the LDA ones are systematically smaller. Another dissociation to a fco phase $\left(I^{\prime}\right)$ is also found, but at a transition pressure higher than that of I-II transition. For both transitions, the values of the scaled volume, lattice constants, and Düsing parameter are quite universal for all three halogens. Band closures are obtained for all three halogen molecular crystals before the transition point from phase I to II.

\section{ACKNOWLEDGMENTS}

M.S. Miao and V.E. Van Doren were supported partly under Grants Nos. G2131.94 and G0347.97 of the Belgian National Science Foundation (NFWO) and partly by the Concerted Action of the University of Antwerpen on "Influence of electron correlation on properties of biomolecules and the performance using density functional theory." J.L. Martins was supported by Grants No. PRAXIS/PCEX/FIS/ 11213/98 and No. POCTI/1999/FIS/36279.
${ }^{1}$ Y. Fujii, K. Hase, Y. Ohishi, H. Fujihisa, N. Hamaya, K. Takemura, O. Shimomura, T. Kikegawa, Y. Amemiya, and T. Matsushita, Phys. Rev. Lett. 63, 536 (1989).

${ }^{2}$ Y. Fujii, K. Hase, N. Hamaya, Y. Ohishi, A. Onodera, O. Shimomura, and K. Takemura, Phys. Rev. Lett. 58, 796 (1987).

${ }^{3}$ K. Takemura, S. Minomura, O. Shimomura, and Y. Fujii, Phys. Rev. Lett. 45, 1881 (1980).

${ }^{4}$ K. Takemura, S. Minomura, O. Shimomura, Y. Fujii, and J.D. Axe, Phys. Rev. B 26, 998 (1982).

${ }^{5}$ Y. Fujii, Y. Ohishi, A. Onodera, K. Takemura, and R.L. Reichlin, Jpn. J. Appl. Phys. 24, 606 (1985).

${ }^{6}$ E.F. Düsing, W.A. Grosshans, and W.B. Holzapfel, J. Phys. C 8, 203 (1984).

${ }^{7}$ D.H. Drickamer, R.W. Lynch, R.L. Clendenen, and E.A. PerezAlbuerne, Solid State Phys. 19, 135 (1966); R.W. Lynch and D.H. Drickamer, J. Chem. Phys. 45, 1020 (1966).

${ }^{8}$ R.W. Lynch and H.G. Drickamer, J. Chem. Phys. 45, 1020 (1966)

${ }^{9}$ R.W. Lynch, R.L. Clendenen, and E.A. Perez-Albuerne, Solid State Phys. 19, 135 (1966).

${ }^{10}$ H. Fujihisa, Y. Fujii, K. Takemura, and O. Shimomura, J. Phys. Chem. Solids 56, 1439 (1995).

${ }^{11}$ Y. Fujii, K. Hase, Y. Ohishi, N. Hamaya, and A. Onodera, Solid State Commun. 59, 85 (1986).

${ }^{12}$ N. Orita, T. Sasaki, and K. Niizeki, Solid State Commun. 64, 391 (1987)
${ }^{13}$ N. Orita, K. Niizeki, and K. Shindo, in 20th International Conference on the Physics of Semiconductors, edited by E. M. Anatassakis and J. D. Joannopoulos (World Scientific, Singapore, 1990), Vol. 3, p. 2201.

${ }^{14}$ H. Sakamoto, M. Shirai, and N. Suzuki, J. Phys. Soc. Jpn. 64, 3860 (1995).

${ }^{15}$ F. Siringo, R. Pucci, and N.H. March, Phys. Rev. B 37, 2491 (1988).

${ }^{16}$ R. Pucci, F. Siringo, and N.H. March, Phys. Rev. B 38, 9517 (1988).

${ }^{17}$ F. Siringo, R. Pucci, and N.H. March, Phys. Rev. B 38, 9567 (1988).

${ }^{18}$ N. Orita, K. Niizeki, K. Shindo, and H. Tanaka, J. Phys. Soc. Jpn. 61, 4502 (1992).

${ }^{19}$ K. Yamaguchi and H. Miyagi, Phys. Rev. B 57, 11141 (1998).

${ }^{20}$ H. Miyagi, K. Yamaguchi, H. Matsuo, and K. Mukose, J. Phys.: Condens. Matter 10, 11203 (1998).

${ }^{21}$ C.H. Park, B.-H. Cheong, K.-H. Lee, and K.J. Chang, Phys. Rev. B 49, 4485 (1994).

${ }^{22}$ I. Souza and J.L. Martins, Phys. Rev. B 55, 8733 (1997).

${ }^{23}$ W.C. Davidon, Math. Program. 9, 1 (1975).

${ }^{24}$ D.M. Ceperley and B.J. Alder, Phys. Rev. Lett. 45, 566 (1980).

${ }^{25}$ J.P. Perdew and A. Zunger, Phys. Rev. B 23, 5048 (1981).

${ }^{26}$ J.P. Perdew and Y. Wang, Phys. Rev. B 45, 13244 (1992).

${ }^{27}$ N. Troullier and J.L. Martins, Phys. Rev. B 43, 1993 (1991). 
${ }^{28}$ R. W. G. Wyckoff, Crystal Structures, 2nd ed. (Interscience, New York, 1963), p. 52.

${ }^{29}$ D. A. Young, Phase Diagrams of the Elements (University of California Press, Berkeley, 1979).

${ }^{30}$ P.R. Vansant, P.E. Van Camp, V.E. Van Doren, and J.L. Martins, Phys. Rev. B 57, 7615 (1998).
${ }^{31}$ B. Montanari and R.O. Jones, Chem. Phys. Lett. 272, 347 (1997).

${ }^{32}$ M.S. Miao, M.-L Zhang, V.E. Van Doren, C. Van Alsenoy, and J.L. Martins, J. Chem. Phys. 115, 11317 (2001).

${ }^{33}$ B.M. Riggleman and H.G. Drickamer, J. Chem. Phys. 38, 2721 (1963). 